\title{
Octogenarian with an untreated femoral neck fracture: upright position during the postoperative course after aortic valve replacement
}

\author{
M. Polastri', L. Di Marco², S. Cantagalli3 , M. Di Eusanio² \\ ${ }^{\top}$ Medical Department of Continuity of Care and Disability, Physical Medicine and Rehabilitation, University \\ Hospital S. Orsola-Malpighi, Bologna; \\ ${ }^{2}$ Department of Cardiac-Thoracic and Vascular Diseases, Cardiac Surgery, \\ University Hospital S. Orsola-Malpighi, Bologna; \\ ${ }^{3}$ Department of Emergency/Urgency, Orthopaedics and Traumatology, \\ University Hospital S. Orsola-Malpighi, Bologna, Italy
}

\section{SUMMARY}

We describe a patient with a femoral neck fracture undergoing an aortic valve replacement.

The study design was a case report. An 82-year-old female with an untreated right femoral neck fracture, and a severe aortic valve stenosis was admitted to a cardiac surgery department for surgical treatment of the valve disease. She underwent aortic valve replacement with a sutureless biological valve prosthesis through a partial sternotomy.

At an early stage, the patient was instructed to make postural changes in the standing position. As a result, she was able to perform body movements associated with either a sitting or standing position.

This case shows that appropriate early mobilization of a patient with a femoral neck fracture is feasible after aortic valve replacement, even though this does not necessarily mean that the patient needs to walk.

Key words: Aortic valve stenosis, Cardiac surgical procedures, Femoral fractures, Octogenarian, Rehabilitation.

Reumatismo, 2015; 67 (1): 26-28

\section{INTRODUCTION}

ctogenarians with aortic valve disease are eligible for valve replacement (1). In the postoperative period, the optimal positioning of the patient in bed and subsequent mobilization are important parts of the rehabilitation process after cardiac surgical procedures (2). However, these steps may be challenging due to the presence of thoracic drains, monitoring/infusion lines, or particular physical conditions. Orthopedic conditions are very uncommon in cardiac surgery patients, especially in the elective setting. On the other hand, femoral fractures require physical therapy in order to preserve the walking ability of patients after surgery (3). Elderly patients who have femoral fractures are more prone to develop cognitive complications during their hospital stay. In general, the mobility of elderly patients after surgically-repaired hip fractures can improve if they participate in the rehabilitation activities (4). Nevertheless, a nonsurgical treatment can be an option when no other treatment possibilities are available or as a temporary solution when other physical conditions require to be treated first. The mortality rate of nonagenarians with hip fractures is approximately $50 \%$ (5). In this report, we describe a patient with an untreated femoral neck fracture undergoing aortic valve replacement.

\section{CASE REPORT}

An 82-year-old female with a body mass index (BMI) of $36.1\left(\mathrm{~kg} / \mathrm{m}^{2}\right)$, an untreated right femoral neck fracture, and severe aortic valve stenosis was admitted to a cardiac surgery department for surgical treatment of a valve disease. She underwent an aor- 
tic valve replacement with a sutureless biological valve prosthesis through a partial sternotomy. The intraoperative and postoperative periods were uneventful. The patient was extubated a few hours after surgery and discharged from the intensive care unit on postoperative day 1. X-ray examination confirmed the presence of an untreated right femoral neck fracture. An ipsilateral endomedullary nail from a previous fracture of the femoral shaft was also present (Figure 1). At the initial physical in-bed evaluation,

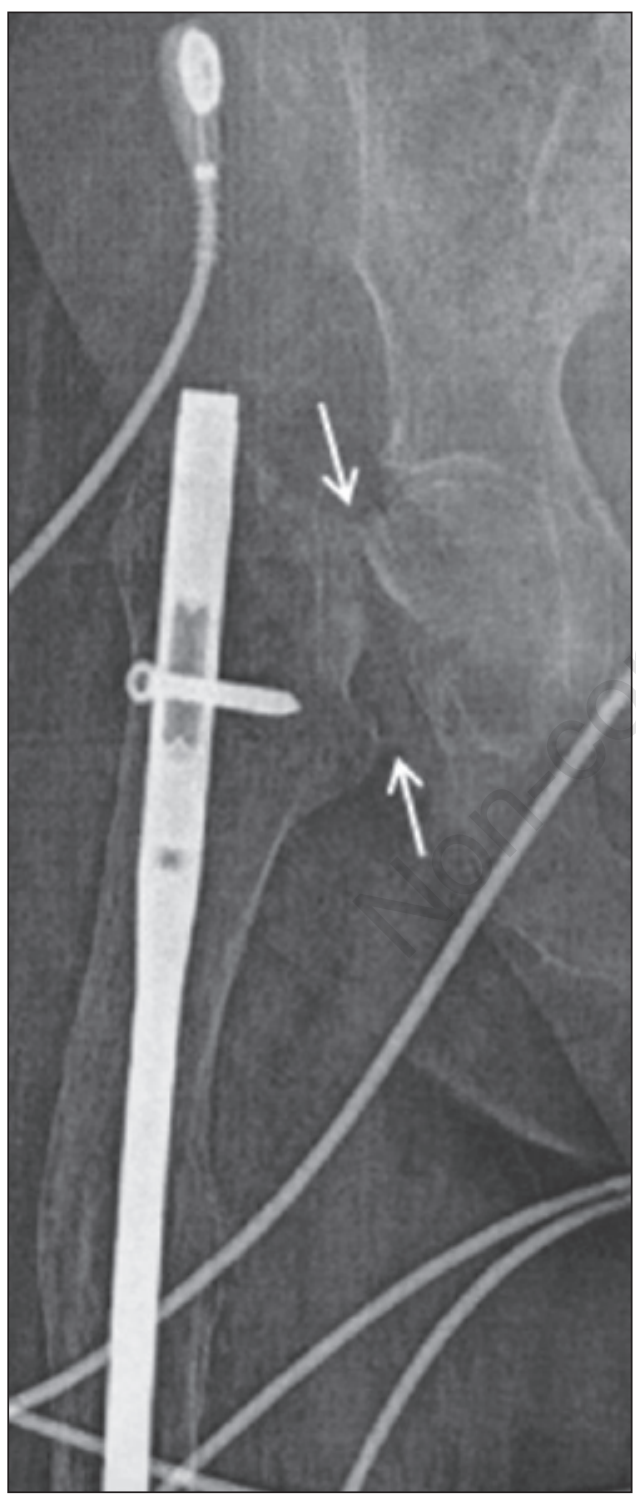

Figure 1 - Fracture of the femoral neck (white arrows). A previous ipsilateral endomedullary nail is also present. the external rotation of the right leg was observed. This finding was consistent with the imaging results. In the first postoperative days, the chest $\mathrm{x}$-rays showed the onset of a bilateral dysventilation (Figure 2). She was prompted to perform respiratory exercises with the goal of maintaining adequate oxygenation and pulmonary function. In the supine position, deep breathing, chest expansion, assisted cough, and chest manual vibration therapy were carried out. Meanwhile she was invited to perform the first in-bed assisted movements. Following a consultation with the orthopedic team, at an early stage, we encouraged her to sit at the edge of the bed to facilitate both feeding and respiratory exercises (incentive spirometer and deep breathings), which were performed at least twice daily during her hospital stay. The patient was lifted off the bed from the right side in order to avoid further fracture site complications. In this way, it was possible to maintain the hip in its natural position. While being moved, the limb was supported until the sitting position was reached. When the patient was confident with these postural changes, which were always assisted, the treatment progressed until she could attain an upright position while keeping the body load on the left limb. The patient was able to sit in a wheelchair that allowed her to use the bathroom. Although walking was not encouraged because of her high BMI,

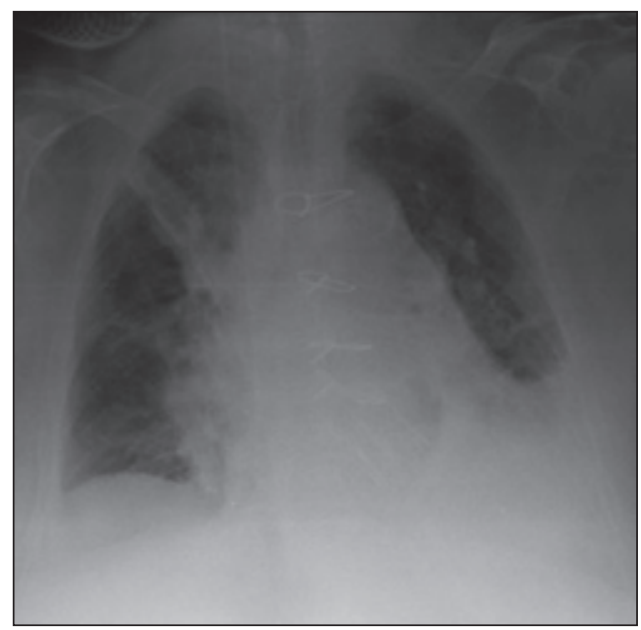

Figure 2 - Bilateral basal dysventilation (more evident in the left lung), and signs of bilateral interstitial edema. 
the patient was instructed to make postural changes at an early stage in the standing position. As a result, she was able to perform body movements associated with either a sitting or standing position. This approach contributed greatly to the patient's final excellent outcome and prevented potential postoperative complications related to age and BMI (i.e., skin ulcers, joint contracture, muscle loss, and immobilization syndrome). The patient was discharged on postoperative day 11 . The patient gave her informed consent to be included in this study.

\section{DISCUSSION AND CONCLUSIONS}

Hip fracture surgical repair in elderly patients with aortic stenosis is associated with an increased risk of morbidity and mortality (6). As previously outlined by our group, in this case an early mobilization contributed to achieve a certain degree of mobility already in the first postoperative days. Being more prone to immobility-related complications, elderly patients may benefit much more than others from mobilization, when appropriate. Moreover, low mobility represents a risk factor if related to hospitalization. In this context, early physical therapy can pave the way for further functional improvement in the postoperative period. In patients who cannot follow a more intensive postoperative rehabilitation program due to their physical conditions, early mobilization and counselling to their caregivers play a key role in their rehabilitation process. Early patient mobilization includes activities such as patient positioning and/or instructions to implement in-bed movements. On the other hand, in-hospital mobility has been found to be directly related to functional decline in a geriatric population (7). Hence our findings seem to be consistent with the notion that early physical therapy can contribute to prevent detrimental conditions in frail elderly patients. The rationale of this study was based on the prevention of postoperative complications that can be potentially dangerous in these complex patients. Indeed this case was further complicated by the fracture site and the patient's BMI. While elderly and obese patients are known to benefit from early rehabilitation, the mobilization program and timing for orthopedic patients undergoing heart surgery remain controversial. In this case, the main challenge was to achieve both the sitting and upright positions in order to avoid worsening the postoperative pulmonary complications. Another advantage of an early mobilization was seen in terms of personal hygiene, as the patient was able to cooperate with the nursing staff, helping them during her hygienic care.

This case shows that, after an aortic valve replacement, it is possible to implement an appropriate early mobilization program for a patient with femoral neck fracture, despite this does not necessarily mean that the patient needs to walk.

\section{REFERENCES}

1. Di Eusanio M, Fortuna D, Cristell D, Pugliese P, Nicolini F, Pacini D, et al. Contemporary outcomes of conventional aortic valve replacement in 638 octogenarians: insights from an Italian Regional Cardiac Surgery Registry (RERIC). Eur J Cardiothorac Surg. 2012; 41: 1247-52.

2. Ridley SC, Heinl-Green A. Surgery for adults. In: Pryor JA, Prasad SA, eds. Physiotherapy for respiratory and cardiac problems, 3rd ed. London: Churcill Livingstone, 2002; 386-7.

3. Al-Ani AN, Flodin L, Söderqvist A, Ackermann $\mathrm{P}$, Samnegård E, Dalén $\mathrm{N}$, et al. Does rehabilitation matter in patients with femoral neck fracture and cognitive impairment? A prospective study of 246 patients. Arch Phys Med Rehabil. 2010; 91: 51-7.

4. Hershkovitz A, Kalandariov Z, Hermush V, Weiss R, Brill S. Factors affecting short-term rehabilitation outcomes of disabled elderly patients with proximal hip fracture. Arch Phys Med Rehabil. 2007; 88: 916-21.

5. Kang BJ, Lee YK, Lee KW, Won SH, Ha YC, Koo KH. Mortality after hip fractures in nonagenarians. J Bone Metab. 2012; 1: 83-6.

6. Adunsky A, Kaplan A, Arad M, Mizrahi EH, Gottlieb S. Aortic stenosis in elderly hip fractured patients. Arch Gerontol Geriatr. 2008; 46: 401-8.

7. Zisberg A, Shadmi E, Gur-Yaish N, Tonkikh O, Sinoff G. Hospital-associated functional decline: the role of hospitalization processes beyond individual risk factors. J Am Geriatr Soc. 2015; 63: 55-62. 